\title{
Epithelial markers in pancreatic carcinoma: immunoperoxidase localisation of DD9, CEA, EMA and CAM 5.2
}

\author{
E Heyderman, S E Larkin, P J O'Donnell, A M R Haines, P J Warren, A Northeast, \\ A G Grant
}

\begin{abstract}
Paraffin wax embedded, formalin fixed sections of 22 adenocarcinomas of the exocrine pancreas were stained with four mouse monoclonal antibodies: DD9-E7, an antibody raised against a human pancreatic tumour xenograft; carcinoembryonic antigen (CEA); epithelial membrane antigen (EMA); and cytokeratin (CAM 5.2). An indirect immunoperoxidase technique without enzyme pre-digestion and an affinitypurified sheep anti-mouse peroxidase conjugate were used. All of the tumours were positive for DD9-E7, EMA, and CAM 5.2. Twenty out of 22 were focally positive for CEA and the staining was often weak. As all of these adenocarcinomas were DD9-E7 positive, absence of staining for DD9-E7 in a tumour makes the diagnosis of adenocarcinoma of the exocrine pancreas very unlikely, and this is of value in distinction from endocrine carcinomas with a marked acinar pattern. The weak CEA staining distinguished pancreatic carcinomas from colorectal tumours. Because the distribution of staining for EMA and CAM 5.2 was no different from that previously seen in adenocarcinomas from other sites, these markers are likely to be of limited value in the differential diagnosis of abdominal adenocarcinomas of uncertain origin.
\end{abstract}

In England and Wales there has been a substantial increase in adenocarcinomas of the exocrine pancreas over the past 75 years. The mortality trebled from an age standardised rate for both sexes of 2.9 per 100000 in the quinquennium 1911-1915 to 10.6 in men and 8.3 in women in $1971-1975 .^{1}$ Since 1974 the mortality has remained fairly stable, with a mean of 2971 a year for men and 2857 for women. ${ }^{2}$ There were slightly more deaths from this cause in men than in women until 1986. Since then the mortality in men has fallen somewhat from 2950 in 1986 to 2905 in 1988 , and risen in women from 3093 to 3103 . $^{3}$ Carcinoma of the pancreas is uncommon under the age of 40 , and the maximum mortality from this cause is at age $65-79 .{ }^{1}$ The prognosis is dismal, with a three year survival of the order of $3 \%$ and very few long term survivors. ${ }^{4}$ Most patients present late, often with metastatic deposits demonstrable at surgery or laparotomy and a resectability rate of only $12 \%{ }^{4}$ The prognosis of cystadenocarcinomas of the pancreas seems to be better than that of solid ductal carcinomas. In a study of 1001 patients, which included ail types of exocrine and endocrine pancreatic carcinomas and ampullary carcinomas, the resectability rate of pancreatic cystadenocarcinomas, which occurred in patients with a mean age of 45 compared with a mean age of 67 for solid tumours, was $67 \%$ (six of nine). The three year survival was $33 \%$ (three of nine), though all three patients finally died of recurrence three, nine, and 16 years later. ${ }^{4}$

The diagnosis of the primary tumour remains difficult for clinicians, while for the histopathologist there are various areas of difficulty. These include (i) determining whether metastatic tumour in nodes, liver, bone, or in other sites could have arisen in the pancreas; (ii) deciding whether an adenocarcinoma in the lung could represent a metastasis from an occult pancreatic carcinoma and therefore be unsuitable for resection; and (iii) distinguishing chronic pancreatitis from well differentiated adenocarcinoma, especially in needle biopsy specimens.

The pronounced desmoplastic response evoked by many pancreatic adenocarcinomas may be of some help in the distinction from other tumours, and many are well differentiated with a rather angular glandular pattern, rather unlike the smoother more ring-like glands seen in gastric or colorectal carcinomas. They are not, however, sufficiently different morphologically from those arising elsewhere in the gastrointestinal tract for a confident diagnosis of a metastatic deposit. In biopsy material origin from other sites cannot be excluded unless there is both a mass in the pancreas felt by the surgeon and pancreatic tissue in the biopsy specimen.

In an initial study DD9-E7 supernatant, raised by immunisation of nude mouse hairy littermates $(\mathrm{nu} /+)$ with a homogenised xenograft of GER pancreatic adenocarcinoma cells, ${ }^{5}$ was used. It had been shown to react strongly with 12 of 14 adenocarcinomas of the exocrine pancreas and with a variety of other normal and neoplastic tissues. Staining of polymorphs and macrophages suggested that the antigen against which DD9-E7 was directed was similar to normal crossreacting antigen, NCA. ${ }^{67}$ Western blot analysis has shown that DD9 recognises a protein epitope on a family of glycoproteins (80-115 kilodaltons) which are distinct from the sialoganglioside and mucin-like pancreatic tumour antigens recognised by other monoclonal 

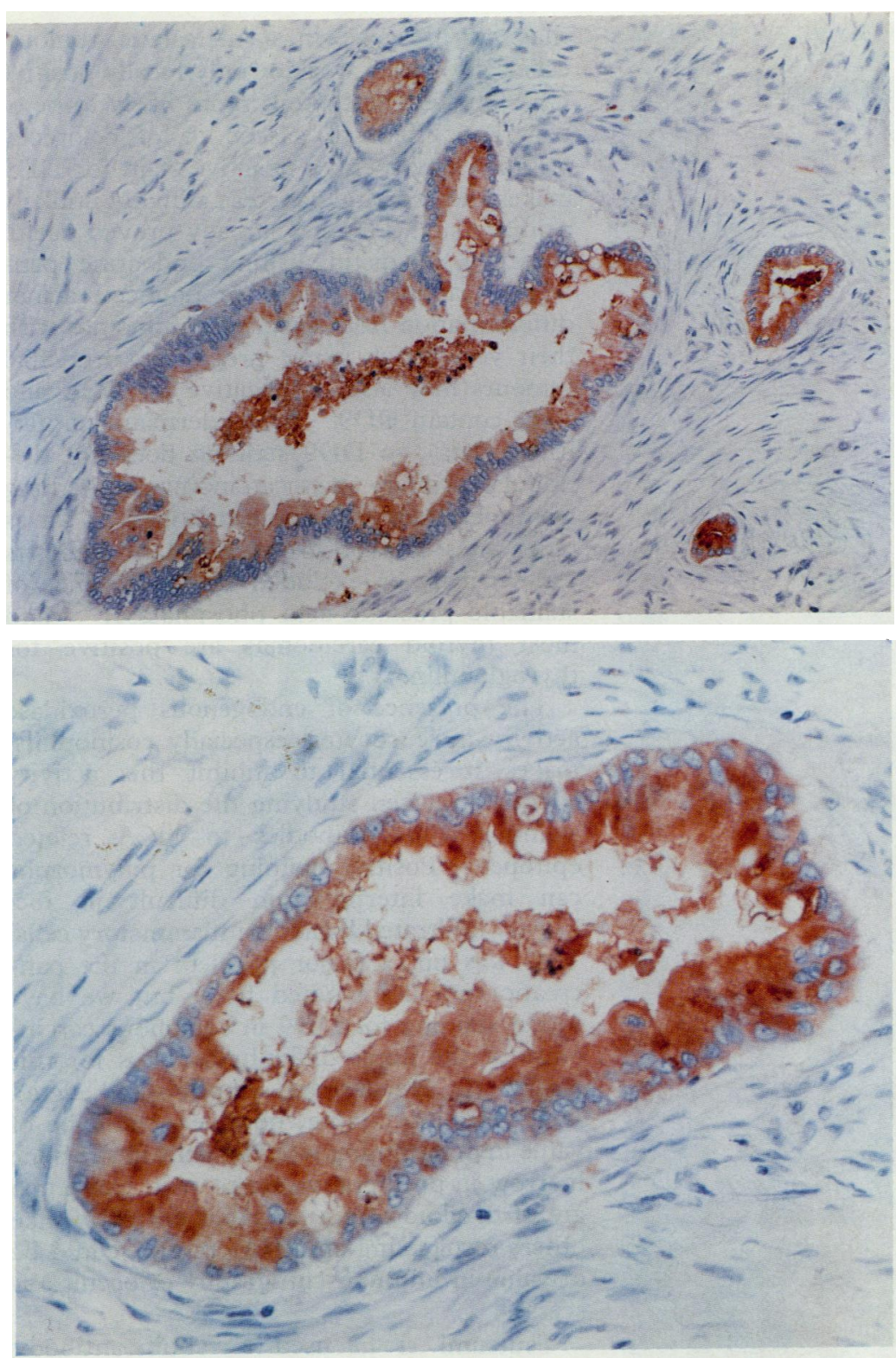

Figure 1 The rather angular acini commonly seen in carcinomas of the exocrine pancreas are seen in this immunostain for DD9.

Figure 2 At higher power the DD9 positive cytoplasmic globules in another malignant acinus are well shown.
(Amersham International plc, Buckinghamshire). It does not stain polymorphs or macrophages and does not seem to recognise an NCA determinant. Anti-EMA was a hybridoma supernatant raised using a preparaton of human milk fat globule membranes ${ }^{1314}$ (Dakopatts Ltd, Buckinghamshire), and CAM $5 \cdot 2$ was also a supernatant, raised by immunisation with the human colon carcinoma cell line HT29. ${ }^{15}$

An indirect immunoperoxidase technique, without enzyme predigestion, and an affinity purified sheep anti-mouse peroxidase conjugate prepared by periodate oxidation ${ }^{16}$ were used. The method was similar to that already published, ${ }^{17}$ except that incubation with the first antibody was carried out overnight instead of for one hour.

The positive controls used in this study were a moderately differentiated colorectal carcinoma for CEA ${ }^{18}$ and CAM $5 \cdot 2,{ }^{15}$ a ductal carcinoma of the breast for EMA, ${ }^{19}$ and a metastatic deposit in the liver of a pancreatic adenocarcinoma for DD9-E7. A monoclonal antibody directed against prostatic acid phosphatase, PASE/4LJ, was used as an irrelevant negative control. This antibody stains some pancreatic islet cells in formalin fixed tissue, but not benign or malignant exocrine pancreatic tissue. ${ }^{20}$ Prostatic chippings showing benign hyperplasia were used as a positive control for PASE/4LJ.

\section{Results}

All of the tumours were positive for DD9-E7, EMA, and cytokeratin, and 20 of 22 were positive for CEA (figs 1-6). Most of the residual pancreatic islets were negative with all four antibodies. A few cells in some islets, however, were positive for cytokeratin, Some positive staining for DD9-E7, EMA, and cytokeratin was found in normal pancreas, as well as in the areas of chronic pancreatitis so frequently found surrounding pancreatic exocrine carcinomas. Although normal pancreatic tissue was negative for CEA, there was positive staining in foci of chronic pancreatitis.

The distribution of staining for DD9 was on the luminal membrane of malignant acini and in the luminal contents (fig 1). Positivity was also often seen as a cytoplasmic globule above the nucleus, separated from the luminal membrane, and corresponding to the position of mucigen granules (fig 2). ${ }^{21}$ The two tumours negative with the supernatant used in the previous study $^{15}$ were positive in this study when ascitic fluid was used. This was likely to be due either to the increase in immunoglobulin content or to prolongation of the first antibody incubation time.

Though some of the tumours were strongly positive for CEA (fig 3), several were weakly or focally positive. Staining was on the luminal membrane or in the cytoplasm, or both, but the globular staining seen with DD9-E7 (fig 2) was not seen with CEA. The normal pancreas surrounding the tumour was 

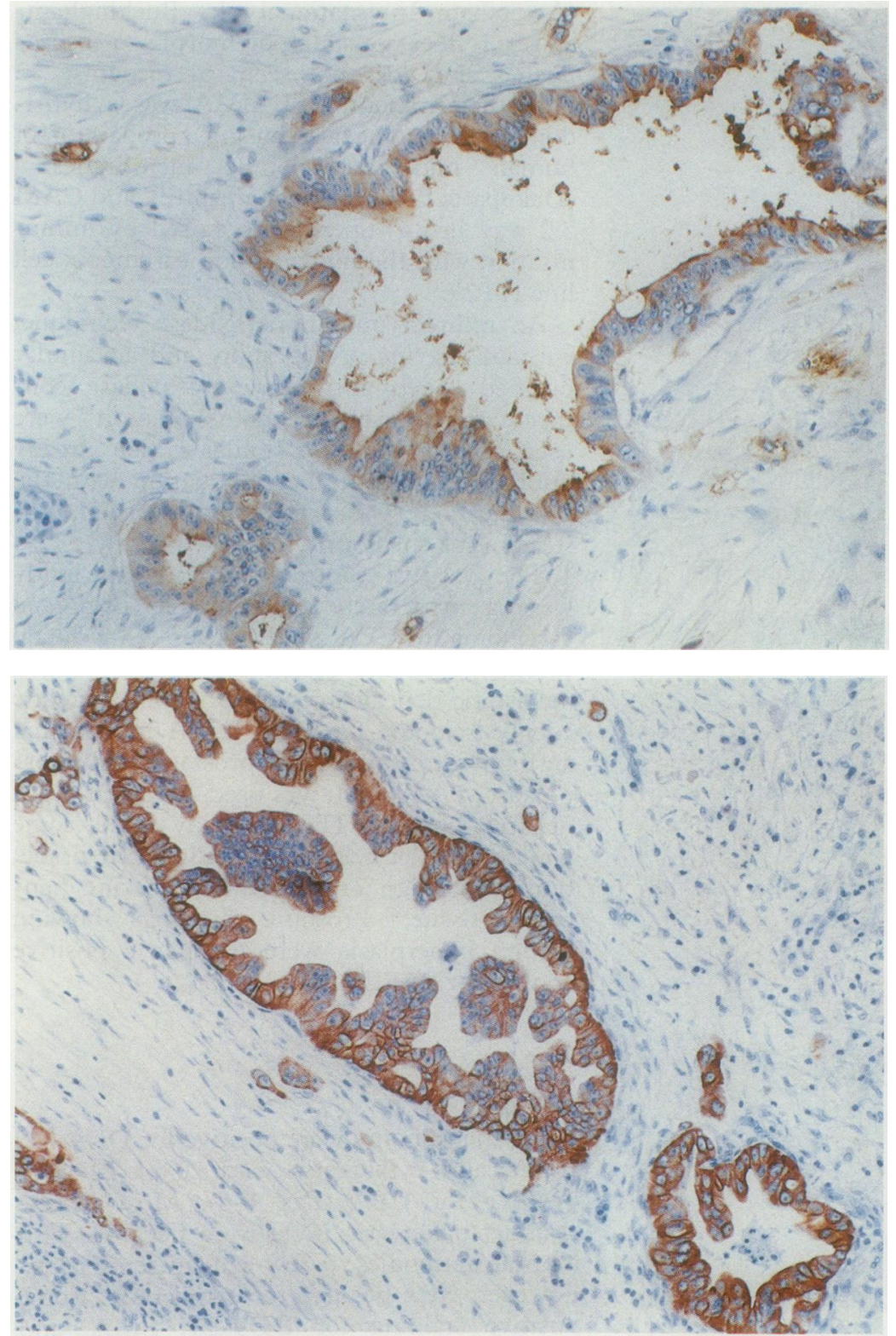

Figure 3 An immunostain for $C E A$ shows malignant acini with a partly luminal, partly cytoplasmic, pattern of staining. A residual islet (top right) is negative.

Figure 4 In this tumour there is a "pericellular" pattern of staining for low molecular weight cytokeratin (CAM 5·2).

negative for CEA, but there were areas of positivity in foci of chronic pancreatitis.

In some tumours CAM $5 \cdot 2$ was localised just under the plasma membrane of the tumour cells, in a "pericellular pattern" (fig 4). In others it was linear and just under the luminal membrane; in the rest it was cytoplasmic. In some tumours the staining was weak and patchy, but in most it was strong. Some residual pancreatic islets showed focal areas of positivity.

The staining for EMA was mainly on the luminal membrane (fig 5), but there was also some cytoplasmic staining (fig 6). In the residual exocrine pancreas intercellular canaliculi similar to those seen in eccrine sweat glands were positive. ${ }^{22}$

\section{Discussion}

The presence of DD9 in all 22 primary pancreatic adenocarcinomas, and its absence in up to $50 \%$ of other adenocarcinomas, ${ }^{12}$ suggests that its presence in a metastatic tumour, especially with a weak or only focally positive CEA, makes the tumour more likely to be of pancreatic than of gastric or of colorectal origin. Conversely, a negative stain for DD9 makes the exocrine pancreas a highly unlikely primary site. This has already proved useful clinically in distinguishing endocrine pancreatic carcinomas with pronounced acinar differentiation from exocrine carcinomas with their very much worse prognosis. Bronchial tumours may also be negative for CEA and some contain DD9 ${ }^{23}$ (E Heydermam, unpublished data), so DD9 staining does not distinguish primary adenocarcinomas of the lung from metastatic pancreatic tumours. As far as other sites are concerned, prostatic deposits can virtually be excluded by the absence of stainable prostatic acid phosphatase, ${ }^{2024}$ and most thyroid carcinomas are positive for thyroglobulin..$^{25} 26$

The presence of endogenous peroxidase activity in leucocytes, especially eosinophils, makes it essential to inhibit this activity adequately when studying the distribution of staining with antibodies to NCA related epitopes. ${ }^{27}$ Positive staining for polymorphs can make interpretation difficult in foci heavily infiltrated by acute inflammatory cells. This was not a major problem in the pancreatic tumours studied here, but we have found staining for DD9 in squamous carcinomas of the lungs difficult to assess for this reason ( $E$ Heyderman, unpublished data). DD9 immunostaining, however, could be of value in determining whether cells in the bone marrow in proliferative states or in leukaemia are of myeloid origin, and this antibody, like others recognising an NCA epitope, could be of value in immunoscintigraphy of occult abscesses.

Tsutsumi et al used a rabbit antibody specifically raised against NCA. They showed the presence of NCA in cryostat sections of pancreatic adenocarcinomas, but not in formalin-fixed, paraffin wax embedded blocks from the same tissues. ${ }^{28}$ All of their tumours, however, were positive with the Dako antiCEA before absorption with a spleen extract. The Dako antibody is well recognised to have NCA activity, and pre-absorption with an NCA preparation is required before it can be used to show CEA and not NCA. ${ }^{29}$ Presumably, the rabbit NCA antibody used by Tsutsumi et al recognises a different, more labile NCA epitope. In another study monoclonal and polyclonal NCA antibodies stained most pancreatic carcinomas and foci of chronic pancreatis. ${ }^{30}$ The monoclonal antibody 374 which recognises a shared CEA, NCA 95, and NCA 55 determinant, stained normal ductal cells and 36 of 40 pancreatic carcinomas. ${ }^{31}$ The monoclonal antibody $47 \mathrm{D} 10$, which was positive in $95 \%$ of pancreatic adenocarcinomas but not in foci of chronic pancreatitis, also stains granulocytes, and may recognise an NCA-related antigen. This antibody, however, is different from DD9 in that it recognises a carbohydrate epitope. ${ }^{32}$ 

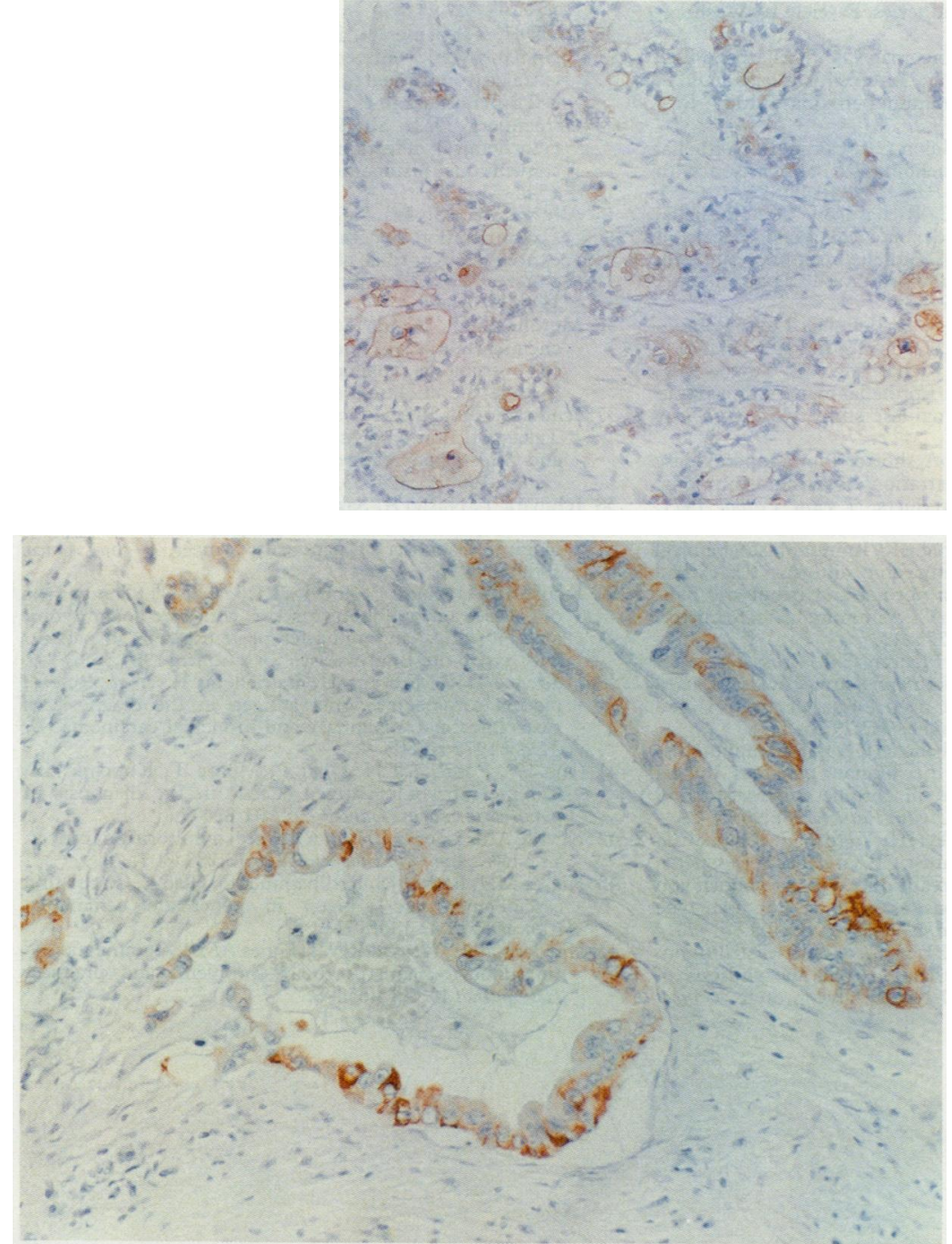

Figure 5 The EMA stain is mainly luminal with the contents of the malignant acini also positive.

Figure 6 In this tumour there is a patchy cytoplasmic positivity for EMA.

There have been several studies on the localisation of CEA in pancreatic carcinomas. ${ }^{28303133-38}$ In the two studies undertaken in 1972 and $1978^{33} 34$ only three out of six and two out of seven of the pancreatic carcinomas were positive, but neither of the antisera used showed positivity in normal colonic mucosa, so that sensitivity was low. More recent studies have shown results very similar to ours, with all or nearly all pancreatic adenocarcinomas showing some CEA. ${ }^{28} 3031$ 35-39

It has been noted in some studies that staining for CEA was stronger in better differentiated pancreatic tumours, ${ }^{3133}$ but this was not notable in our series, nor in those of others. ${ }^{28}$ The weakness or focal distribution of staining was surprising. In a recent study serum concentrations of CEA were increased in $91 \%$ of patients with pancreatic cancer ${ }^{39}$ and in most cases in earlier studies. ${ }^{40-43}$ The weak staining, compared with that in our colon carcinoma control, may reflect low levels of storage of CEA, because immunocytochemistry can only detect stored material and does not necessarily accurately reflect synthesis. ${ }^{44}$

The absence of CEA staining in normal pancreas led us to hope that its demonstration could be of value in the differentiation of tumour from chronic pancreatitis, but, as found by others, ${ }^{31}{ }^{35}$ both adenocarcinomas and foci of chronic pancreatitis were positive, though such foci were found to be negative with the F6 CEA antibody. ${ }^{30}$

EMA and cytokeratin, while of possible value for tumour immunolocalisation in patients where specificity may not be required, do not distinguish pancreatic adenocarcinomas from those arising elsewhere.

We are very grateful to Ms CA Makin and Amersham International plc for their generous gifts of CAM $5 \cdot 2$ and antiCEA, respectively. The work was supported by St Thomas's Hospital Research Endowment Fund (AMRH) and the Cancer Research Campaign (SEL and AGG).

The photomicrographs were taken using Ektor Gold 25.

1 Office of Population Censuses and Surveys-Cancer Research Campaign. Cancer Statistics. Incidence, survival and mortality in England and Wales. Studies in medical and population subjects. London: HMSO, 1981 : No 43.

population subjects. London: HMSO, 1981:No 43 .
2 Office of Population Censuses and Surveys. Mortality Statistics. Causes. London: HMSO, Series DH2 19771989:Nos 1-14.

3 Office of Population Censuses and Surveys. Mortality Statistics. Causes. London: HMSO, Series DH2 1990; No 15.

4 Connolly MM, Dawson PJ, Michelassi F, Moossa AR, Lowenstein F. Survival in 1001 patients with carcinoma of the pancreas. Ann Surg 1987;206:366-73.

5 Grant AG, Duke D, Hermon-Taylor J. Establishment and characterization of primary human pancreatic carcinoma in continuous cell culture and in nude mice. $B r J$ Cancer 1979;39:143-51.

6 Mach J-P, Putztaszeri G. Carcinoembryonic antigen (CEA): Demonstration of a partial identity between CEA and a normal glycoprotein. Immunochemistry 1972;9:1031-4.

7 Von Kleist $S$, Chavanel G, Burtin B. Identification of a normal antigen that cross-reacts with the carcinoembryonic antigen. Proc Natl Acad Sci USA 1972;69: 2492-4.

8 Atkinson BF, Ernst CS, Herlyn M, Steplewski Z, Sears HF, Koprowski $H$. Gastrointestinal cancer-associated antigen in immunoperoxidase assay. Cancer Res 1982;42:4820-3.

9 Borowitz MJ, Tuck FL, Sindelar WF, Fernstein PD, Metzgar RS. Monoclonal antibodies against human panMetzgar RS. Monoclonal antibodies against human panantigen on glandular epithelia and adenocarcinomas. antigen on glandular ep
$J N C I$ 1984;72:999-1005.

10 Nilsson O, Mansson J-E, Brezicka T, et al. Fycosyl-Gml-a ganglioside associated with small cell lung carcinomas. Glycoconjugate 1984;1:43-9.

11 Burtin P, Chavanel G, Hirsch-Marie H. Characterization of a second normal antigen that cross-reacts with CEA J Immunol 1973;111:1926-8.

12 Grant AG, Harris PM, Heyderman E, Larkin SE, Pym B, Hermon-Taylor J. Production of monoclonal antibodies against pancreatic exocrine cancer: a study of six different immunisation regimes. Br J Cancer 1985;52:543-50.

13 Cordell J, Richardson TC, Pulford KAF, et al. Production of monoclonal antibodies against human epithelial membrane antigen for use in diagnostic immunocytochemistry. brane antigen for use in diagno

14 Heyderman E, Strudley I, Powell G, Richardson TC, Cordell JL, Mason DY. A new monoclonal antibody to epithelial membrane antigen (EMA)-E29. A comparison of its immunocytochemical reactivity with polyclonal antiEMA antibodies and with another monoclonal antibody,
HMFG-2. Br J Cancer 1985;52:355-61.

15 Makin CA, Bobrow LG, Bodmer WF. Monoclonal antibody to cytokeratin for use in routine histopathology. $J$ Clin Pathol 1984;37:975-83.

16 Nakane PK, Kawaio A. Peroxidase-labelled antibody: a new method of conjugation. J Histochem Cytochem 1974;22. method of

17 Heyderman E. Tumour markers. In: Polak JM, Noorden S, eds. Immunocytochemistry: Practical applications in pathology and biology. 2nd ed. Bristol: John Wright and Sons Ltd, 1986:502-32.

18 Gold P, Freedman SO. Demonstration of tumor-specific antigens in human colonic carcinomata by immunological tolerance and absorption techniques. J Exp Med 1965; 121:439-62.

19 Heyderman E, Steele K, Ormerod MG. A new antigen on the epithelial membrane: its immunoperoxidase localisation in normal and neoplastic tissue. J Clin Pathol 1979;32:35-9.

20 Haines AMR, Larkin SE, Richardson AP, Stirling RW, Heyderman E. Production of a novel hybridoma antibody (PASE/4L J) to human prostatic acid phosphatase. Br J

21 Cubilla AL, Fitzgerald PJ. Tumors of the exocrine pancreas. 
Atlas of tumor pathology. 2nd series, Fascicle 19. Washington: AFIP, 1984:125.

22 Graham RM, McKee PH, Chapman DV, Richardson TC, Stokoe MR, Heyderman E. Intercellular canaliculi in eccrine sweat glands. An immunoperoxidase study. $\mathrm{Br} J$ Dermatol;85:112:397-403.

23 Heyderman E, Larkin SE, Makin CA, Corrin B. Epithelial markers in pleural mesotheliomas. A comparison with primary lung carcinoma. J Pathol 1985;145:81A.

24 Heyderman E, Brown BME, Richardson TC. Epithelial markers in prostatic, bladder and colorectal cancer: an immunoperoxidase study of epithelial membrane antigen, carcinembryonic antigen and prostatic acid phosphatase. J Clin Pathol 1984;37:1363-9.

25 Lo Gerfo P, Li Volsi V, Colaccio D, Feind C. Thyroglobulin production by thyroid cancers. $J$ Surg Res $1978 ; 24: 1-6$.

26 Wilson NW, Pambakian H, Richardson TC, Stokoe MR, Makin CA, Heyderman E. Epithelial markers in thyroid carcinoma: an immunoperoxidase study. Histopathology 1986;10:815-29.

27 Heyderman E, Neville AM. A shorter immunoperoxidase technique for the demonstration of carcinoembryonic antigen and other cell products. J Clin Pathol 1977; 30:138-40.

28 Tsusumi $Y$, Nagura H, Watanabe K. Immunohistochemical observations of carcinoembryonic antigen (CEA) and CEA-related substances in normal and neoplastic pancreas. Pitfalls and caveats in CEA immunohistochemistry. Am J Clin Pathol 1984;82:535-42.

29 Nap M, Klaske A, Hoor T, Fleuren G-J. Cross-reactivity with normal antigens in commercial anti-CEA sera, used for immunohistology. The need for tissue controls and absorptions. Am J Clin Pathol 1983;79:25-31.

30 Albers GH, Fleuren G, Escribano MJ, Nap M. Immunohistochemistry of CEA in the human pancreas during development, in the adult, in chronic pancreatitis and pancreatic carcinoma. Am J Clin Pathol 1988;90:17-22.

31 Batge B, Bosslet K, Sedlacek HH, Kern HF, Kloppel G. Monoclonal antibodies against CEA-related components discriminate between pancreatic duct type carcinomas and discriminate between pancreatic duct type carcinomas and
non-neoplastic duct lesions as well as nonduct type non-neoplastic duct lesions as well as nonduct type
lesions. Virchows Arch (Pathol Anat) 1986;408:361-74.

32 Ho MK, Kato KP, Durda PJ, et al. Tissue distribution, immunochemical characterization, and biosynthesis of $47 \mathrm{D} 10$, a tumor associated surface glycoprotein. Cancer
Res 1987;47:241-50.

33 Denk H, Tappeiner G, Eckerstorfer R, Holzner JH. Carcinoembryonic antigen (CEA) in gastrointestinal and extrastrointestinal tumors and its relationship to tumorcell differentiation. Int J Cancer 1972;10:262-72.

34 Goldenberg DM, Sharkey RM, Primus FJ. Immunocytochemical detection of carcinoembryonic antigen in conventional histopathology specimens. Cancer 1978;42: $1546-53$.

35 Allum WH, Stokes HJ, Macdonald F, Fielding JWL. Demonstration of carcinoembryonic antigen (CEA) expression in normal, chronically inflamed, and malignant pancreatic tissue by immunohistochemistry. J Clin Pathol 1986;39:610-4

36 Morohoshi T, Kanda M, Horie A, et al. Immunocytochemical markers of uncommon pancreatic tumors: acinar chemical markers of uncommon pancreatic tumors: acinar cell carcinoma, pancreatoblastoma, and solid

37 Ichihara T, Nagura $H$, Nakao A, Sakamoto J, Watanabe $T$ chihara T, Nagura H, Nakao A, Sakamoto J, Watanabe T,
Takagi H. Immunohistochemical localisation of CA 19-9 and CEA in pancreatic carcinoma and associated diseases. Cancer 1988;61:324-33.

38 Ichihara T, Nakao A, Sakamoto J, et al. Application of the immunoperoxidase method for rapid intraoperative path ological diagnosis of pancreatic cancer. J Surg Oncol 1989;40:8-16.

39 Kuusela P, Haglund P, Roberts PJ, Jalanko H. Comparison of CA-50, a new tumour marker, with carcinoembryonic antigen (CEA) and alpha-fetoprotein (AFP) in patients antigen (CEA) and alpha-fetoprotein (AFP) in patients

40 Laurence DJR, Stevens U, Bettleheim R, et al. Role of plasma carcinoembryonic antigen in the diagnosis of gastrointestinal, mammary and bronchial carcinoma. $\mathrm{Br}$ Med J 1972;iii:605-9.

41 Ona FV, Zamcheck N, Dhar P, Moore T, Kupchik HZ Carcinoembryonic antigen (CEA) in the diagnosis of pancreatic cancer. Cancer 1973;31:324-7.

42 Neville AM, Cooper EH. Biochemical monitoring of cancer: a review. Ann Clin Biochem 1976;13:283-305.

43 Kalser MH, Barkin JS, Redlhammer D, Heal A. Circulating carcinoembryonic antigen in pancreatic carcinoma. Carcinoembryonic antigen

44 Heyderman E, Warren PJW, Haines AMR. CommentaryImmunocytochemistry today-problems and practice. Histopathol 1989;15:653-8. 